\title{
Size Rate of an Alternative Aggregation Petri net developed under a Modular Approach
}

\author{
Juan-Ignacio Latorre-Biel $^{1} \quad$ Emilio Jiménez-Macías $^{2} \quad$ Julio Blanco $^{2} \quad$ Mercedes Perez $^{2}$ \\ ${ }^{1}$ Department of Mechanical Energetic and Materials Engineering, Public University of Navarre, Spain, \\ juanignacio.latorre@unavarra.es \\ ${ }^{2}$ High Technical School of Industrial Engineering, University of La Rioja, Spain, \\ \{emilio.jimenez, julio.blanco, mercedes.perez\}@unirioja.es
}

\begin{abstract}
Petri nets allow describing formal models of discrete event systems, which might show counterintuitive behaviors. The design of a discrete event system, composed by known subsystems, requires the definition of the interrelations between them. This feature can be modeled in the structure of the Petri net by arcs and link transitions. The choice of the best configuration might be a hard problem to solve due to the foreseeable combinatorial explosion. In order to alleviate the computer resources required for exploring the different feasible combinations of the subnets, a single model with exclusive entities can be developed by an alternatives aggregation Petri net. In this paper the construction of such a model with four subnets and certain precedence constrain is discussed. Also, a reduction in the size of the amount of required information for describing the alternative structural configurations is calculated for different sizes of the subnets.
\end{abstract}

Keywords: Petri nets, modelling and simulation, modular design, alternative structural configuration, decision support system

\section{Introduction}

Petri nets constitute a paradigm in the modeling of discrete event systems (Silva, 1993). The simplicity of its rules, the double representation of a model, both graphical and matrix-based, as well as its ability to describe features, such as parallelism, precedence, concurrence, synchronization, or competence for shared resources, makes Petri nets an invaluable tool for applications such as performance evaluation or structural analysis (David and Alla, 2005).

One fruitful application of Petri nets is the field of decision making support (Latorre et al, 2014c). However, other methodologies can be considered for this task (Bruzzone and Longo, 2010). Among them, modeling and simulation have been applied successfully (Jiménez-Macías and Pérez-Parte, 2004; Piera et al, 2004; Longo et al, 2013; Mújica et al, 2010). In particular, Petri nets can be used for quasi-optimal operation or design of complex systems (Latorre et al, 2014b). In particular, a design process usually requires choosing between different alternative structural configurations, which makes this kind of decision problem singular in nature (Latorre and Jiménez, 2013).

Some particular problems of design are tackled by the selection of certain subsystems, such as particular machines, manufacturing lines, or even manufacturing facilities, and the ulterior choice of the way, these systems are related. These decisions involve the definition of the interchange of information, parts, products, vehicles, persons, or whatever the flow between subsystems is. Moreover, these decisions configure the behavior of the subsystems by features such as precedence, synchronization, or parallelism (Latorre et al, 2014a).

A classic methodology to address this decision problem starts considering a different model, or alternative Petri net, for every alternative structural configuration of the system (Latorre et al, 2014c). This approach, however, presents some drawbacks, such as the need to develop a large number of models and to analyze specifically every one of them, or to discard good decisions by reasons, such as intuition, rough analysis, personal preferences, lack of awareness, etc. Another important drawback is the large amount of data required for representing all the alternative Petri nets, since they may be created by different combinations of the shared subnets (Latorre-Biel et al, 2015).

This combinatorial process for constructing feasible solutions of the design problem, implies the fact that many data required to represent a set of alternative Petri nets is redundant: every shared Petri net belongs to many models and its description is repeated every time, increasing in this way the size of the description of the system with alternative structural configurations (w/ASC) (Latorre et al, 2014b).

Moreover, this is not the only type of redundant information, present in a set of alternative Petri nets, since a given transition between subnets or link transitions, can also be present in several alternative Petri nets (Latorre-Biel et al, 2015).

In order to overcome these drawbacks, a family of Petri net-based formalisms has been developed. All of 
them can model a discrete event system w/ASC. The exclusiveness of the alternative structural configurations is represented by a set of exclusive entities. In particular, the alternatives aggregation Petri nets can integrate in a natural way a set of shared subnets (Latorre et al, 2013). This paper deals with the design process of a discrete event system, once a set of subsystems has been chosen. Another constrain of the problem is a relation of strict precedence of one of the subnets, and the other three.

The rest of the paper is organized as follows. Section 2 defines the formalism that will be considered for developing the model of the system: the AAPN. Section 3 states in a detailed way the design problem to be solved. The three following sections (Section 4 , Section 5 and Section 6) present different types of relations between the subsystems and provide with the expressions to calculate the size rate of the AAPN. Section 7 addresses the complete AAPN model, representing the cases discussed in the previous sections, compares the size rates, and comments the trend of the size rate of all the cases as the size of the shared subnets grow. Finally, the conclusions derived from this piece of research are stated in Section 8.

\section{Alternatives Aggregation Petri Nets}

An AAPN is a formalism that contains a set of exclusive entities. On the other hand, a set of exclusive entities is a collection of mathematical elements representing the exclusiveness that characterizes the alternative structural configurations of the modeled system, i.e. only one of them can be chosen as a result of a decision. In particular, the exclusive entities in an AAPN are the so called choice variables, Boolean variables that configure the guard functions of certain link transitions in the model.

An AAPN is defined by a set of subnets, some of which are shared by different alternative Petri nets, another set of link transitions between the subnets, and a last set of guard functions of choice variables associated to some link transitions.

Alternatives aggregation Petri nets (AAPN) can be applied successfully in the modeling process of discrete event systems with alternative structural configurations (w/ASC), where the different configurations present common or shared subnets.

In this case, the number of redundant information in the form of shared subnets that can be removed in the AAPN may be significant. Shared subnets contain places and transitions and their removal contribute to a reduction in the size of the incidence matrix of the AAPN, resulting, for example, in a speed up of the simulation of the net, useful for performance analysis.

The construction process of an AAPN from a set of alternative Petri net is quite straightforward. The following steps can be followed to achieve this objective: a) Decompose the alternative Petri nets into subnets and link transitions. The election of the limits of every subnet is a choice of the modeler. However, certain criteria can be considered, such as associating each subnet with a physical element of the real system or trying to guarantee that every subnet is shared by the largest number of alternative Petri nets.

b) Take one of the alternative Petri nets as seed of the AAPN. Associate the first choice variable to every link transition.

c) Consider the following alternative Petri net (ith alternative Petri net) and compare it to the seed of the $A A P N$. Every subnet of the alternative Petri net that does not belong to the AAPN should be added to the $A A P N$. Add to the AAPN all the link transtions of the alternative Petri net. Every added link transition should be associated to the ith choice variable.

d) Apply reduction rules to the quasi-identical link transitions of the AAPN.

e) Repeat steps c) and d) until all the alternative Petri nets have been added to the seed of the AAPN and the final AAPN model is complete.

With these considerations as background, the following section will define the scope of the design problem to be solved.

\section{Statement of the problem}

The objective of this paper is to show the feasibility, the methodology, and some advantages of constructing an AAPN for decision making support in the design process of a kind of discrete event system.

In particular, the system to be designed should include a single unit of every one of four different subnets $\left\{R_{A}, R_{B}, R_{C}, R_{D}\right\}$. These subnets are chosen to have only an input and an output link transitions. In addition, the input transition presents a single output place. Analogously, the output transition has a single input place.

Moreover, one of the subnets should comply with a relation of strict precedence with the other three subnets, which should evolve in parallel, simultaneously and/or alternatively.

Moreover, solutions with other precedence relations between the subnets, leading to an unbounded Petri net, or presenting deadlocks, should be discarded.

Complying with the mentioned constraints, three options with different types of relations between the subnets are considered and analyzed in the following sections.

The following notation will be considered:

$A_{r}, B_{r}, C_{r}, D_{r}$, are the number of rows of the incidence matrix of the alternative Petri nets $R_{A}, R_{B}, R_{C}, R_{D}$ respectively.

$A_{c}, B_{c}, C_{c}, D_{c}$, are the number of columns of the incidence matrix of the alternative Petri nets $R_{A}, R_{B}, R_{C}$, $R_{D}$ respectively. 


\section{Case I}

The first structure for the solution of the decision problem has been drawn in Figure 1, where a general structure of an alternative Petri net has been represented. In this figure, the four different subnets are depicted by means of clouds, which is an informal or incomplete description of a Petri net, since the input and output places of the link transitions, belonging to every subnet, are not specified.

Furthermore, the internal structure of the subnet is not detailed, due to the fact that the purpose of this representation is to develop a general description, where the relationship between the subnets is pointed out. The link transitions are depicted explicitly in the representation of Figure 1.

In this figure, the different subnets are represented by a general name of $R_{w}, R_{x}, R_{y}, R_{z}$, since all the combinations of the shared subnets $\left\{R_{A}, R_{B}, R_{C}, R_{D}\right\}$ can be substituted in the positions of $\left\{R_{w}, R_{x}, R_{y}, R_{z}\right\}$ defining feasible solutions for the discrete event system to be designed. In fact, there are four feasible combinations of subnets in this case 1 , leading to four alternative Petri nets $\left\{R_{1}, R_{2}, R_{3}, R_{4}\right\}$. Two of them are depicted in Figure 2.

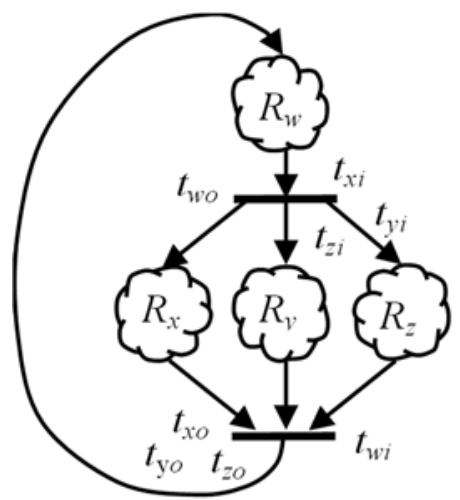

Figure 1. Structure of the link transitions for the case 1 as solution of the discrete event system to be designed.

It can be seen that the link transitions can be named after their input or output subnets. Since the four subnets are connected to each link transition, any of the link transitions can have four different names, as it can be seen in Figure 2.
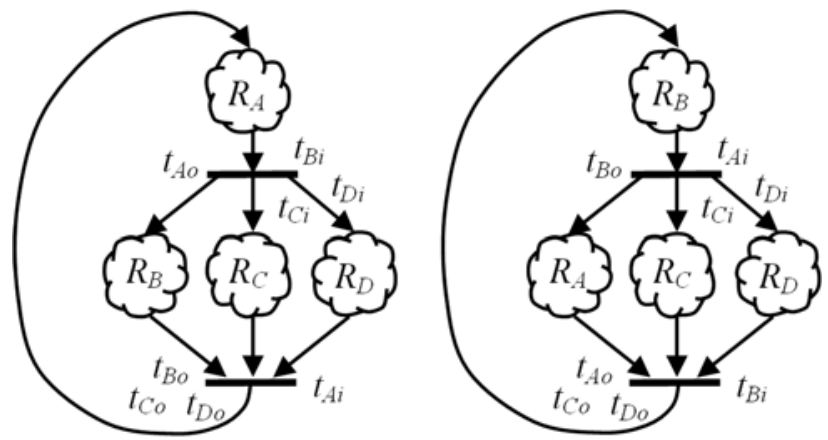

Figure 1. Two feasible solutions of case I: $R_{1 a}$ and $R_{2 a}$.
Following the steps detailed in section 2, it is possible to construct a single alternatives aggregation Petri net from the complete set of four alternative Petri nets. This AAPN contains the four subnets $\left\{R_{A}, R_{B}, R_{C}, R_{D}\right\}$, as well as two link transitions from every alternative Petri net $\left\{R_{1 a}, R_{2 a}, R_{3 a}, R_{4 a}\right\}$. Due to the fact that it is not possible to find quasi-identical transitions among the link transitions of the AAPN, it is not possible to apply a reduction rule to diminish the number of link transitions. For this reason, the number of link transitions of the AAPN is $2 \cdot 4=8$.

Let us consider the following notation:

$r$ and $c$ are the number of rows and columns of an alternative Petri net respectively.

$r$ ' and $c$ ' are the number of rows and columns of the resulting AAPN respectively.

It has to be considered that the size of the four alternative Petri nets $\left\{R_{1 a}, R_{2 a}, R_{3 a}, R_{4 a}\right\}$ is the same and can be calculated as follows:

$$
\begin{aligned}
& r=A_{r}+B_{r}+C_{r}+D_{r} \\
& c=A_{c}+B_{c}+C_{c}+D_{c}+2
\end{aligned}
$$

where the number 2 added to the calculation of $c$ comes from the two link transitions of every alternative Petri net $\left\{R_{1 a}, R_{2 a}, R_{3 a}, R_{4 a}\right\}$.

Moreover, the size of the incidence matrix of the AAPN is:

$$
\begin{aligned}
& r^{\prime}=A_{r}+B_{r}+C_{r}+D_{r} \\
& c^{\prime}=A_{c}+B_{c}+C_{c}+D_{c}+8
\end{aligned}
$$

Let us call $x=A_{c}+B_{c}+C_{c}+D_{c}$; hence,

$$
\begin{aligned}
& c=x+2 \\
& C^{\prime}=x+8
\end{aligned}
$$

It is possible to calculate the reduction size of the AAPN, when compared with the set of alternative Petri nets $S_{R}=\left\{R_{1 a}, R_{2 a}, R_{3 a}, R_{4 a}\right\}$. Both of them, the AAPN and $S_{R}$ represent the same system and contain exactly the same information. However, the amount of data required by any of them is quite different:

$$
\text { size rate }=\operatorname{size}(\mathrm{AAPN}) / \operatorname{size}\left(S_{R}\right)=
$$$$
=r^{\prime} \cdot c^{\prime} /\left(4 \cdot r^{\prime} \cdot c\right)
$$

According to (1) and (2), it is possible to state that

$$
r=r^{\prime}=A_{r}+B_{r}+C_{r}+D_{r}
$$

as a consequence

$$
\text { size rate }=c^{\prime} /(4 \cdot c)
$$

Moreover, considering (3) it is obtained that

$$
\text { size rate }=(x+8) /[4 \cdot(x+2)]
$$

Figure 3 , represents the trend of the size rate for different values of $x=A_{c}+B_{c}+C_{c}+D_{c}$, which is the addition of the number of places belonging to every shared subnet. In particular, it can be seen that as $x$ increases, the size rate decreases to a limit value given by:

$$
\text { size rate }=(1+8 / x) /[4 \cdot(1+2 / x)]
$$

Calculating the limit of the previous expression as $x$ increases to infinity is $1 / 4=0.25$.

This means that as the number of places in the shared subnets grows, the size of the AAPN approaches to $25 \%$ 
of the size of the original set of alternative PN $S_{R}$. In other words, with a $25 \%$ of the data, the AAPN provides the same modeling information than the set $S_{R}$.

The axis of abscissas in Figure 3 has been represented with a logarithmic scale with the purpose of detailing the trend for small sizes of the shared subnets.

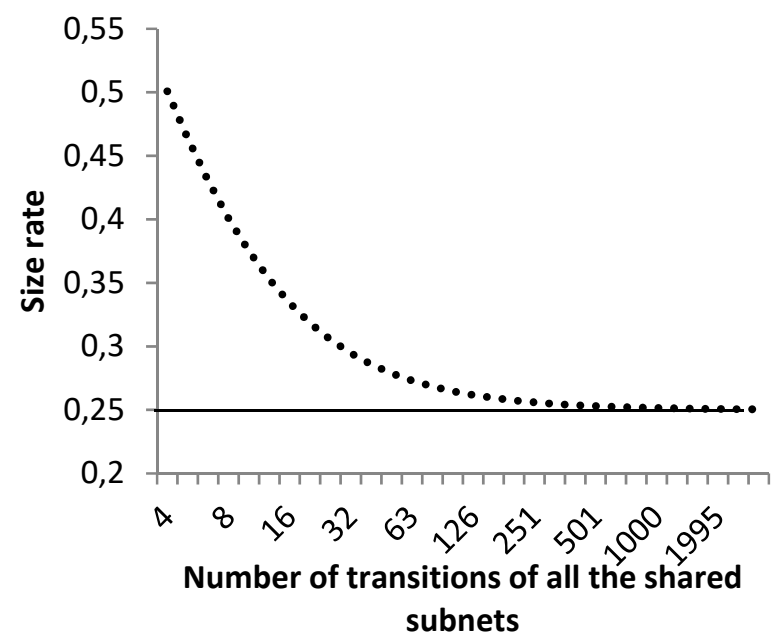

Figure 3. Size rate of and AAPN representing a complete set of alternative Petri nets in case I.

\section{Case II}

This second case corresponds to another structure for the link transitions in the construction of alternative Petri nets that verify the constraints of the problem stated in section 3. Figure 4 depicts two examples of the four feasible combinations of the shared subnets for constructing such alternative Petri nets.

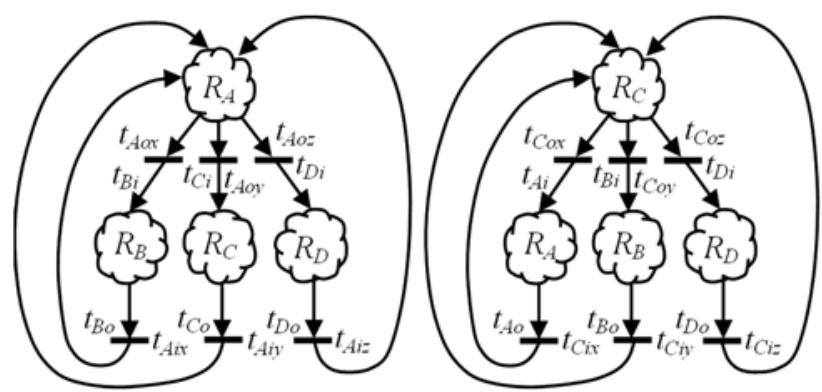

Figure 4. Two feasible solutions of case II: $R_{1}$ (left) and $R_{3}$ (right).

The four subnets $\left\{R_{A}, R_{B}, R_{C}, R_{D}\right\}$ can be combined in four different ways for constructing four alternative Petri nets $S_{R b}=\left\{R_{1 b}, R_{2 b}, R_{3 b}, R_{4 b}\right\}$. Every one of these alternative Petri nets presents 6 link transitions. Moreover, the application of the steps for constructing an AAPN, described in section 2, allow obtaining a single Petri net, representing the complete set of alternative Petri nets $S_{R b}$.

The resulting AAPN presents 24 link transitions. However, every link transition has a quasi-identical transition in this set. It is possible to find a couple of examples in Figure 4. In particular, transition $t_{C i}$ of $R_{1}$ is quasi-identical to $t_{C i x}$ in $\mathrm{R}_{3}$. Moreover $t_{C o}$ in $R_{1}$ is quasiidentical to $t_{C o x}$ in $R_{3}$. In fact, these transitions are not identical due to the fact that in the AAPN, a different choice variable is associated to each transition from every couple of quasi-identical transitions.

As a consequence of the previous considerations 12 quasi-identical transitions of the AAPN can be combined with their quasi-identical counterparts, leading a Petri net with only 12 link transitions from the original 24 (6 from every original alternative Petri net).

It has to be considered that the size of incidence matrices of the four alternative Petri nets $\left\{R_{1 b}, R_{2 b}, R_{3 b}\right.$, $\left.R_{4 b}\right\}$ is the same, $r \cdot c$, where:

$$
\begin{aligned}
& r=A_{r}+B_{r}+C_{r}+D_{r} \\
& c=A_{c}+B_{c}+C_{c}+D_{c}+6
\end{aligned}
$$

where 6 is the number of link transitions of any of the original alternative Petri nets $\left\{R_{1 b}, R_{2 b}, R_{3 b}, R_{4 b}\right\}$.

Analogously, the size of the incidence matrix of the AAPN is $r^{\prime} \cdot c^{\prime}$, where:

$$
\begin{aligned}
& r^{\prime}=A_{r}+B_{r}+C_{r}+D_{r} \\
& c^{\prime}=A_{c}+B_{c}+C_{c}+D_{c}+12
\end{aligned}
$$

Let us call $x=A_{c}+B_{c}+C_{c}+D_{c}$; hence,

$$
\begin{aligned}
& c=x+6 \\
& c^{\prime}=x+12
\end{aligned}
$$

The reduction size of the AAPN is: size rate $=$ size $($ AAPN $) /$ size $\left(S_{R b}\right)=r^{\prime} \cdot c^{\prime} /(4 \cdot r \cdot c)$

According to (9) and (10), it is possible to state that

$$
r=r^{\prime}=A_{r}+B_{r}+C_{r}+D_{r}
$$

as a consequence size rate $=c^{\prime} /(4 \cdot c)$

Moreover, considering (11) it is obtained that size rate $=(x+12) /[4 \cdot(x+6)]$

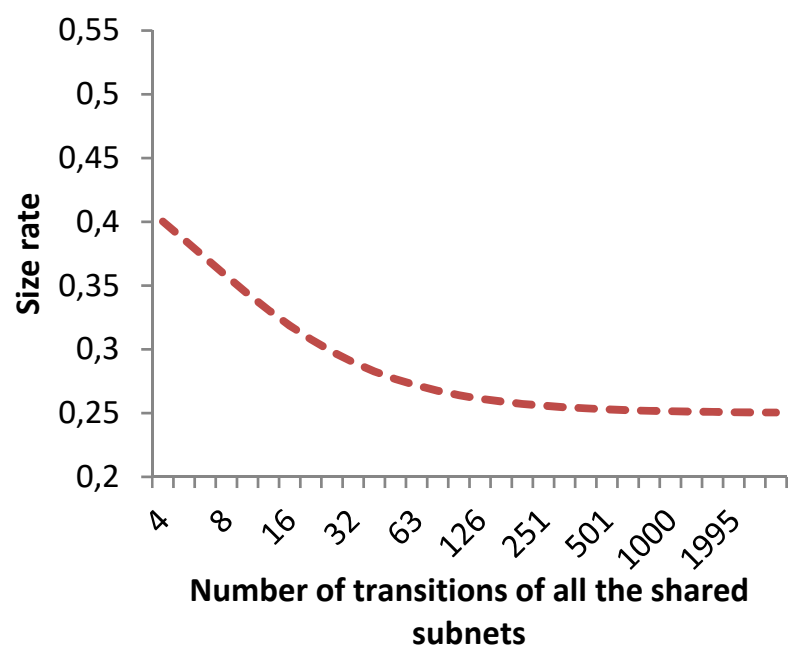

Figure 5. Size rate of and AAPN representing a complete set of alternative Petri nets in case II.

Figure 5 represents the trend of the size rate for different values of $x=A_{c}+B_{c}+C_{c}+D_{c}$, which is the addition of the number of places belonging to every shared subnet. In particular, it can be seen that as $x$ increases, the size rate decreases to a limit value given by:

$$
\text { size rate }=(1+12 / x) /[4 \cdot(1+6 / x)]
$$


Calculating the limit of the previous expression as $x$ increases to infinity is $1 / 4=0.25$, the same as in case I. However, the curve that represents the size rate in case I is different to the one representing the same parameter in case II due to a different function of $x$ : (7) versus (14). In fact, the size rate corresponding to case II is smaller than the size rate of case I.

Figure 5 represents the trend of the size rate of the AAPN obtained for case II.

\section{Case III}

A different structure for the link transitions in the construction of alternative Petri nets that verify the constraints of the problem stated in section 3 is presented in this case III. Figure 6 represents two examples of the twelve feasible combinations of the shared subnets for constructing such alternative Petri nets.
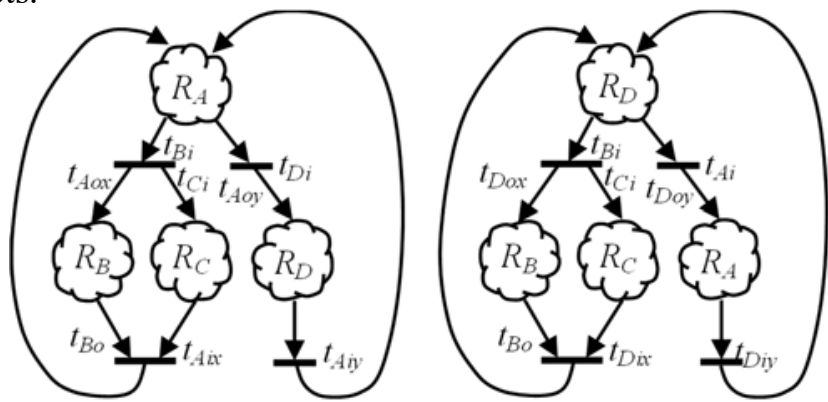

Figure 6. Two feasible solutions of case III: $R_{1}$ (left) and $R_{12}$ (right).

In case III The four subnets $\left\{R_{A}, R_{B}, R_{C}, R_{D}\right\}$ can be combined in twelve different ways for constructing twelve alternative Petri nets $S_{R c}=\left\{R_{1 c}, R_{2 c}, \ldots, R_{12 c}\right\}$. Every alternative Petri net of $S_{R c}$ contains 4 link transitions. Furthermore, it is possible to apply to $S_{R c}$ the steps mentioned in section 2 for obtaining and equivalent AAPN.

As a result, an AAPN with 48 link transitions can be obtained. In this Petro net 12 couples of quasi-identical transitions can be found. Just to give two examples that have been depicted in Figure 6, it is possible to consider transition $t_{D i}$ of $R_{1}$ is quasi-identical to $t_{D i y}$ in $\mathrm{R}_{12}$. Moreover $t_{\text {Aiy }}$ in $R_{1}$ is quasi-identical to $t_{A i}$ in $R_{12}$.

As a consequence of the previous considerations 12 quasi-identical transitions of the AAPN can be combined with their quasi-identical counterparts, leading a Petri net with only 36 link transitions from the original 48 (4 from every original alternative Petri net).

The incidence matrices' size of $S_{R c}=\left\{R_{1 c}, R_{2 c}, \ldots\right.$, $\left.R_{12 c}\right\}$ is:

$$
\begin{aligned}
& r=A_{r}+B_{r}+C_{r}+D_{r} \\
& c=A_{c}+B_{c}+C_{c}+D_{c}+4
\end{aligned}
$$

where 4 is the number of link transitions of any of the original alternative Petri nets $S_{R c}=\left\{R_{1 c}, R_{2 c}, \ldots, R_{12 c}\right\}$.

Analogously, the size of the incidence matrix of the AAPN is $r^{\prime} \cdot c^{\prime}$, where:

$$
\begin{aligned}
& r^{\prime}=A_{r}+B_{r}+C_{r}+D_{r} \\
& c^{\prime}=A_{c}+B_{c}+C_{c}+D_{c}+36
\end{aligned}
$$

Let us call $x=A_{c}+B_{c}+C_{c}+D_{c}$; hence,

$$
\begin{aligned}
& c=x+4 \\
& c^{\prime}=x+36
\end{aligned}
$$

The reduction size of the AAPN is:

$$
\begin{aligned}
& \text { size rate }=\text { size }(\text { AAPN }) / \operatorname{size}\left(S_{R b}\right)= \\
& =r^{\prime} \cdot c^{\prime} /(4 \cdot r \cdot c)
\end{aligned}
$$

According to (16) and (17), it is possible to state that

$$
r=r^{\prime}=A_{r}+B_{r}+C_{r}+D_{r}
$$

as a consequence

$$
\text { size rate }=c^{\prime} /(12 \cdot c)
$$

Moreover, considering (18) it is obtained that size rate $=(x+36) /[12 \cdot(x+4)]$

Figure 7 , represents the trend of the size rate for different values of $x$. In particular, it can be seen that as $x$ increases, the size rate decreases to a limit value given by

$$
\text { size rate }=(1+36 / x) /[12 \cdot(1+4 / x)]
$$

Calculating the limit of the previous expression as $x$ increases to infinity is $1 / 12=0.0833$.

Figure 7 represents the trend of the size rate of the AAPN obtained for case III.

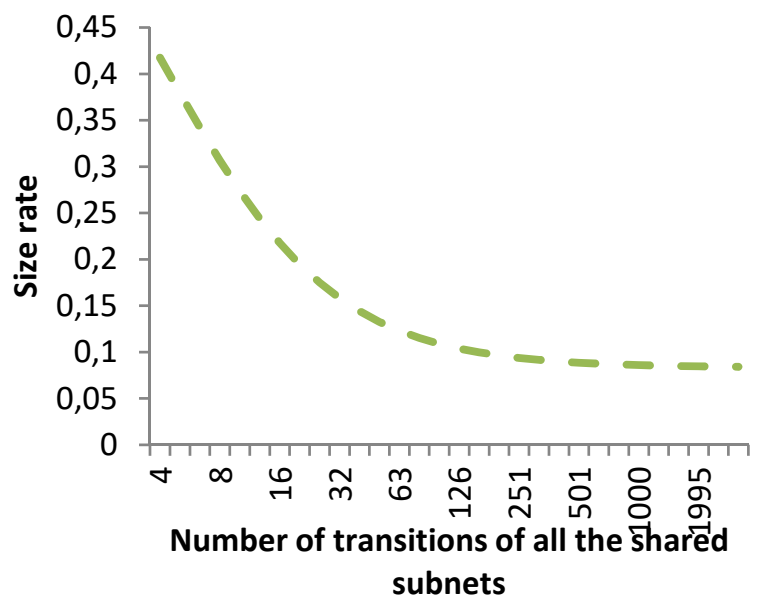

Figure 7. Size rate of and AAPN representing a complete set of alternative Petri nets in case III.

\section{Complete model}

The steps for constructing an AAPN can also be applied to all the alternative Petri nets in the sets $S_{R a}, S_{R b}$, and $S_{R c}$, defined in the previous sections.

As a result, a single AAPN can be developed to represent the 20 alternative Petri nets defined by the sets $S_{R a}, S_{R b}$, and $S_{R c}$. The size rate of this AAPN, compared to the original sets of alternative Petri nets can be defined as follows:

$$
\begin{aligned}
& \text { size rate }=\operatorname{size}(\mathrm{AAPN}) /\left[\operatorname{size}\left(S_{R a}\right)+\operatorname{size}\left(S_{R b}\right)+\right. \\
& \left.+\operatorname{size}\left(S_{R c}\right)\right]=\left(r^{\prime} \cdot c^{\prime}\right) /[4 \cdot r \cdot(x+2)+ \\
& +4 \cdot r \cdot(x+6)+12 \cdot r \cdot(x+4)]
\end{aligned}
$$

where $r^{\prime}=r$; hence,

size rate $=c^{\prime} /[4 \cdot(x+2)+4 \cdot(x+6)+12 \cdot(x+4)]$

$c^{\prime}=x+8+12+36-12$ 
In this last expression, the added numbers correspond to the link transitions provided to the global AAPN by the partial AAPN constructed from $S_{R a}, S_{R b}$, and $S_{R c}$ respectively. The negative number corresponds to quasiidentical transitions. As a result:

$$
\text { size rate }=(x+44) /(20 \cdot x+80)
$$

Calculating the limit of the previous expression as $x$ increases to infinity is $1 / 20=0.05$.

Figure 8 represents the trend of the size rate of the AAPN obtained for the global AAPN

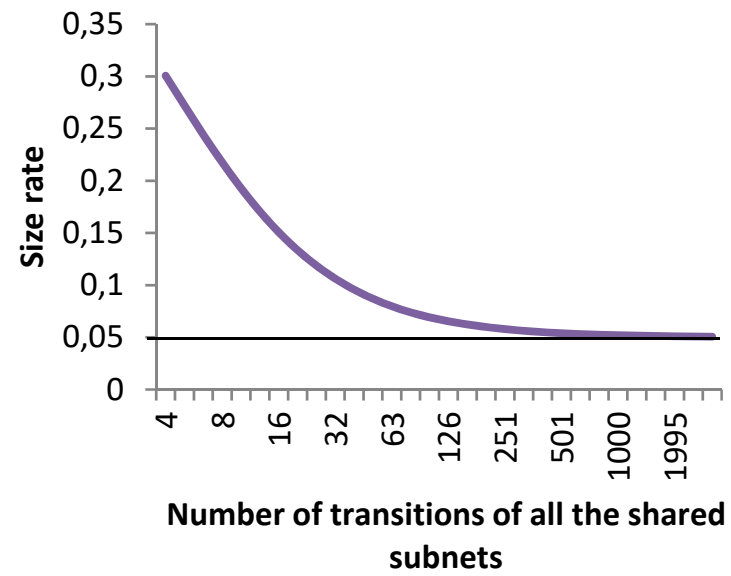

Figure 8. Size rate of global AAPN representing 20 alternative Petri nets.

As an example of the power of reduction of the size of the model achieved by an AAPN, it can be considered that, for example, if the addition of the number of transitions of the four subnets is 200 , the size rate of the AAPN that corresponds to the complete model is 0.06 . This value means that the size of the AAPN is only $6 \%$ of the size of the complete set of 20 alternative Petri nets, despite the fact that the amount of useful information is the same in both models. In other words, $94 \%$ of the information contained in the set of 20 alternative $\mathrm{PN}$ can be removed to alleviate the computational effort for simulation.

\section{Conclusions}

The modular construction of a Petri net model of a system has been discussed. This concept has useful application in searching for feasible solutions for a design process of a discrete event system with alternative structural configurations.

The use of an appropriate formalism, such as the AAPN, allows reducing significantly the size of the model that represents all the feasible solutions of the design problem. In the case study presented in this paper, a minimal size rate of $5 \%$ can be obtained by the global model, when compared to the original alternative Petri nets. In particular, in the mentioned case study, $95 \%$ of the data of the original models is removed in the AAPN. However, the useful information contained in the AAPN is exactly the same as in the original model.
The impact of this research on discrete event systems can be summarized by the fact that in certain cases the design of a system might be developed much faster by a modular construction of alternative solutions and the simulation of compact Petri net models.

\section{References}

A.G. Bruzzone and F. Longo. An advanced system for supporting the decision process within large-scale retail stores. Simulation-Transactions of the Society for Modeling and Simulation International, 86:742-762, 2010.

R. David and H. Alla. Discrete, Continuous and Hybrid Petri Nets. Berlin: Springer, 2005.

E. Jiménez-Macías, and M. Pérez-Parte. Simulation and optimization of logistic and production systems using discrete and continuous Petri nets. Simulation, 80(3):143$152,2004$.

J.I. Latorre, E. Jiménez, J. Blanco, and J. C. Sáenz. Optimal Design of an Olive Oil Mill by Means of the Simulation of a Petri Net Model. International Journal of Food Engineering, 10(4):573-582, 2014a.

J.I. Latorre, E. Jiménez, and M. Pérez. The optimization problem based on alternatives aggregation Petri nets as models for industrial discrete event systems. Simulation, 89(3):346-361, 2013.

J.I. Latorre, E. Jiménez, M. de la Parte, J. Blanco, and E. Martínez. Control of Discrete Event Systems by Means of Discrete Optimization and Disjunctive Colored PNs: Application to Manufacturing Facilities. Abstract and Applied Analysis, 2014 Article ID 821707:1-16, 2014b.

J.I. Latorre, E. Jiménez, and M. Pérez. Sequence of decisions on discrete event systems modeled by Petri nets with structural alternative configurations. Journal of Computational Science, 5(3):387-394, 2014c.

J.I. Latorre and E. Jiménez. Simulation-based optimization of discrete event systems with alternative structural configurations using distributed computation and the Petri net paradigm. Simulation, 89(11):1310-1334, 2013.

J. I. Latorre-Biel, E. Jiménez Macías, J. L. García-Alcaraz, J. C. Sáenz-Díez Muro, M. Pérez de la Parte. Alternatives aggregation petri nets applied to modular models of discrete event systems. In Proceedings of the European Modelling and Simulation Symposium. EMSS 2015, pages 465-470. Bergeggi. Italy, 2015.

F. Longo, L. Nicoletti, A. Chiurco, A. O. Solis, M. Massei, and R. Diaz. Investigating the behavior of a shop order manuf-acturing system by using simulation. SpringSim EAIA 2013

M. A. Mújica, M.A. Piera, and M. Narciso. Revisiting state space exploration of timed coloured Petri net models to optimize manufacturing system's performance. Simulation Modelling Practice Theory, 18:1225-1241, 2010.

M.À. Piera, M. Narciso, A. Guasch, and D. Riera. Optimization of logistic and manufacturing system through simulation: A colored Petri net-based methodology. Simulation, 80(3):121-129, 2004.

M. Silva. Introducing Petri nets, F. Di Cesare, editor, Practice of Petri Nets in Manufacturing, pages 1-62. Chapman \& Hall. 1993. 